\title{
Finding Zeros of Quartic Polynomials by Using Radicals
}

\author{
Sureyya Sahin \\ sahin508@gmail.com
}

June 24, 2018

\begin{abstract}
We present a technique for finding roots of a quartic general polynomial equation of a single variable by using radicals. The solution of quartic polynomial equations requires knowledge of lower degree polynomial equations; therefore, we study solving polynomial equations of degree less than four as well. We present self-reciprocal polynomials as a specialization and additionally solve a numerical example.
\end{abstract}

\section{Introduction}

Solving polynomial equations have been a central topic not only for its usage in pure mathematics but also in physical applications. Consequently, solution of polynomial equations attracted interest peaking at the nineteenth century from mathematicians, which lead to development of modern algebra.

As we are mostly familiar with, a polynomial in a single variable is an expression involving powers of the variable multiplied by some coefficients. Thus, we obtain a polynomial equation when we equate a polynomial to zero. Solution of a polynomial equation is essentially finding values of the variable such that the evaluation of the polynomial at that specific value of the variable gives us zero. These specific values are called the zeros of the polynomial [1], or roots of the polynomial equation. We define a general [2] (or generic [1]) polynomial as the one whose coefficients are open variables but not numerical values. A polynomial is named a quartic polynomial when the degree of the polynomial, that is, the highest power of the polynomial is four. Similarly, a cubic polynomial has the highest degree three while a quadratic polynomial has the highest degree two.

Based on these elementary definitions, we state that our study will be on solving a general single variable quartic polynomial equation by radicals. By radicals, we mean expressions which are a combination of the sums, differences, quotients, products as well as the roots greater than or equal to two of the coefficients of the polynomial [1]. Even though it is standard to learn solving quadratic polynomial equations by using radicals in the contemporary curriculum, we don't observe a common interest in learning how to solve cubic and quartic equations by such a method. In engineering and applied sciences literature, we meet other techniques for solving quartic polynomial equations; yet, we know that a general polynomial of degree four (or less) can be solved by radicals, which gives us an exact result with ease of implementation, and also has the advantage of evolving to maturity within the past centuries. Therefore, an understanding of finding roots of a general quartic polynomial equation by radicals would lead us an adequate means to solve problems in which these equations occur.

Thus, in Section 2, we discuss some background material including a theorem which provides the number of zeros of a general polynomial. In Section 3, we discuss solution of the quartic polynomial, starting by Tschinhaus transformation [1] and obtaining the resolvent poly- 
nomial equation, which is necessary in the solution procedure. The resolvent polynomial necessary to solve a general quartic polynomial equation is cubic; thus, we discuss solving cubic polynomial equations in Section 3.1. In Section 3.2, we proceed solving the quartic polynomial and give the formulas necessary for solving by radicals. As a case study, we will discuss solving self-reciprocal quartic polynomials by radicals in Section 4. Self-reciprocal polynomials occur in applications which require inversion in a circle. Such an application is available in [3]. We give a numerical example in Sections 4.1.

\section{Number of Roots}

Before formulating the solution procedure to quartic polynomial equations, we discuss the number of roots of a polynomial equation. As we will see in Section 3, the transformations necessary for finding the zeros of a polynomial can lead us to the conclusion that a polynomial of degree $n$ can have more than $n$ zeros. In this section, we show that this kind of conclusion is incorrect for a polynomial whose coefficients belong to a subfield $(F)$ of the complex number field $(\mathbb{C})$.

Before dealing with number of roots, we introduce and discuss the quartic equations. A general quartic polynomial equation in a single variable is written in the form:

$$
a_{4} x^{4}+a_{3} x^{3}+a_{2} x^{2}+a_{1} x+a_{0}=0
$$

with $a_{i} \in F, i=0, \ldots, 4$. Additionally, the field $F$ contains the field of rational numbers $(\mathbb{Q})$. The left side of the equation 1 can be represented as a polynomial $p(x)$, which belong to a polynomial ring $F[x]$. Consequently, a polynomial ring contains any polynomial of degree $n$ with coefficients in $F$. The rest of the discussion is developed in the literature for any polynomial of degree $n$, thus valid for an arbitrary polynomial in $F[x]$.

We will let the zeros of a polynomial be an element of $\mathbb{C}$ even though the polynomial itself is an element of the polynomial ring $F[x]$. This lets us to take advantage of the fundamental theorem of algebra, which we present from [4] without proof. The proof can be found in texts such as [4].

Theorem 1 (Fundamental Theorem of Algebra). The field of complex numbers is algebraically closed; that is, every polynomial in $\mathbb{C}[x]$ has a zero in $\mathbb{C}$.

Thus, for any zero $x_{i} \in \mathbb{C}$ of a polynomial $p(x)$, we can factor as $p(x)=\left(x-x_{i}\right) q(x)$ with $q(x) \in \mathbb{C}[x]$. The consequence of the fundamental theorem of algebra on the number of roots of a polynomial equation of degree $n$ is presented in the following theorem

Theorem 2. If $p(x) \in F[x]$ be a polynomial of degree $n$ in the field $F$, which is a subfield of $\mathbb{C}$, then $p(x)$ has exactly $n$ zeros in $\mathbb{C}$.

Proof. If we take a polynomial $p(x)$ of degree $n$, by the fundamental theorem of algebra, we must have a linear factor $x_{1} \in \mathbb{C}$ so that $p(x)=$ $\left(x-x_{1}\right) q(x)$ with $p\left(x_{1}\right)=0$. But $q(x)$, which is of degree $n-1$ must also have a linear factor by the fundamental theorem of algebra. Thus, $q(x)=\left(x-x_{2}\right) r(x)$ with $q\left(x_{2}\right)=0$ and $x_{2}$ not necessarily different from $x_{1}$. The polynomial $p(x)$ can be written as $p(x)=\left(x-x_{1}\right)\left(x-x_{2}\right) r(x)$ where $r(x)$ has degree $n-2$. Continuing this procedure recursively leads us to completely split the polynomial into linear factors $x_{1}, x_{2}, \ldots, x_{n}$ such that

$$
p(x)=\left(x-x_{1}\right)\left(x-x_{2}\right) \cdots\left(x-x_{n}\right)
$$

Thus, we conclude that a polynomial of degree $n$ has $n$ zeros in the algebraically closed field $\mathbb{C}$.

Using Theorem 2, we can conclude that the quartic polynomial in Eq. 1 has four zeros in $\mathbb{C}$. Obviously, if the zeros we seek are located in a subset instead of $\mathbb{C}$, the number of zeros that we are interested in can be less than four.

\section{General Quartic Polynomial of a Single Variable}

Obtaining the number of roots of the general quartic polynomial equation in $F[x]$, we can proceed 
with finding its roots. The procedure we offer is based on a method outlined in [4], which is a variation of Ferrari's solution. This method requires solving a resolvent cubic polynomial equation. Thus, in Section 3.1, we discuss finding the zeros of a cubic polynomial before we obtain the roots of the general quartic equation in Section 3.2 .

We start by considering the Eq. 1 of Section 2. The polynomial equation being quartic makes it necessary that $a_{4} \neq 0$, otherwise we would end up an equation of degree not equal to four. Using this fact, we can manipulate Eq. 1 to make the coefficient of the fourth power term one, that is, we can transform into a monic quartic polynomial equation. Thus, dividing both sides of the equation 1 , we obtain:

$$
x^{4}+b_{3} x^{3}+b_{2} x^{2}+b_{1} x+b_{0}=0
$$

with $b_{i}=a_{i} / a_{4}, i=0, \ldots, 3$, so $b_{i} \in F$. We use Eq. 2 for the rest of the discussion without losing generality.

We proceed by applying Tschirnhaus transformation; thus, we let $y=x+b_{3} / 4$ so that $x=$ $y-b_{3} / 4$. Substituting for $x$ in Eq. 2, we obtain the depressed [4] quartic polynomial equation as

$$
y^{4}+q y^{2}+r y+s=0
$$

where

$$
\begin{aligned}
q & =-\frac{3}{8} b_{3}^{2}+b_{2} \\
r & =\frac{1}{8} b_{3}^{3}-\frac{1}{2} b_{3} b_{2}+b_{1} \\
s & =-\frac{3}{256} b_{3}^{4}+\frac{1}{16} b_{3}^{2} b_{2}-\frac{1}{4} b_{3} b_{1}+b_{0}
\end{aligned}
$$

We now consider writing the polynomial equation in terms of sums of squares. To achieve this, we first arrange the depressed quartic polynomial equation as

$$
y^{4}=-q y^{2}-r y-s
$$

We will consider the case that the right side of Eq. 5 is not a square in which case the solution would reduce to solving quadratic polynomial equations without further effort. Thus, we proceed by introducing a variable $z$ and complete the left side to square as

$$
\left(y^{2}+\frac{1}{2} z\right)^{2}=-q y^{2}-r y-s+z y^{2}+\frac{1}{4} z^{2}
$$

We would like to complete the right side of Eq. 6 to a square, i.e. to a form $(m y+k)^{2}$, as well. For this reason, we collect the terms of the right hand side of Eq. 6 in the variable $y$ to obtain

$$
(z-q) y^{2}-r y+\left(\frac{1}{4} z^{2}-s\right)
$$

Thus, we let

$$
m=\sqrt{z-q}
$$

Then, to complete Eq. 7 to a square in the form $(m y+k)^{2}$, we must have $2 m k=-r$. Therefore, we find

$$
k=-\frac{r}{2 m}=-\frac{r}{2 \sqrt{z-q}}
$$

Thus, the equation 7 becomes

$$
(m y+k)^{2}-\frac{r^{2}}{4(z-q)}+\left(\frac{1}{4} z^{2}-s\right)
$$

When Eq. 10 is a complete square, we must have

$$
-\frac{r^{2}}{4(z-q)}+\left(\frac{1}{4} z^{2}-s\right)=0
$$

Obviously, if Eq. 11 holds, then we have the square $(m y+k)^{2}$. Therefore, we need to solve Eq. 11 to proceed finding the zeros of the quartic polynomial. Since $z$ is an open variable, we can assume $z \neq q$. Then, we can multiply both sides of Eq. 11 by $4(z-q)$ and expand the numerator to obtain

$$
z^{3}-q z^{2}-4 s z+\left(4 q s-r^{2}\right)=0
$$

Hence, solving Eq. 12 is a necessary step for obtaining the roots of the quartic polynomial equation by radicals. Consequently, we call the cubic polynomial in Eq. 12 the resolvent of the general quartic polynomial equation. We discuss solution of the resolvent cubic polynomial in section 3.1. 


\subsection{Solving the Resolvent Polynomial}

In this section, we solve the resolvent cubic polynomial, which is Eq. 12. We can make the Tschinhaus transformation for the cubic polynomial; thus, we let $t=z-q / 3$ so that $y=t+q / 3$. Substituting into Eq. 12, we obtain the depressed resolvent cubic polynomial equation as

$$
t^{3}+g t+h=0
$$

where

$$
\begin{aligned}
g & =-\frac{1}{3} q^{2}-4 s \\
h & =-\frac{2}{27} q^{3}+\frac{8}{3} q s-r^{2}
\end{aligned}
$$

We make one more transformation [4] for $t=$ $u-g /(3 u)$ with $u$ being another open variable, then substitute into Eq. 13. Collecting the terms of the resulting equation under a common denominator and considering that the numerator of the equation is zero, we obtain

$$
u^{6}+h u^{3}-\frac{g^{3}}{27}=0
$$

Thus, we obtain a quadratic equation in $u^{3}$. Completing Eq. 15 to a square and solving for $u^{3}$, we obtain

$$
\begin{aligned}
& u_{1}^{3}=-\frac{h}{2}-\sqrt{\frac{h^{2}}{4}+\frac{g^{3}}{27}} \\
& u_{2}^{3}=-\frac{h}{2}+\sqrt{\frac{h^{2}}{4}+\frac{g^{3}}{27}}
\end{aligned}
$$

We will take an interlude and discuss finding the roots of polynomial equations in the form $x^{3}-a_{0}=0$ in $\mathbb{C}[x]$. We know that any complex number $a$ can be written in the form $a=r(\cos \theta+$ $i \sin \theta$ ). As a consequence of De Moivre's theorem [5], we can write

$$
a^{n}=r^{n}(\cos (n \theta)+\mathrm{i} \sin (n \theta))
$$

Thus, if we write $a_{0}$ in the form [3] $r \operatorname{cis}(\theta)=$ $r(\cos \theta+\mathrm{i} \sin \theta)$, and let $n=1 / 3$, we obtain the three cube roots $x$ as

$$
\begin{aligned}
x & =\sqrt[3]{r} \operatorname{cis}\left(\frac{\theta+2 j \pi}{3}\right) \\
& =\sqrt[3]{r} \operatorname{cis}\left(\frac{2 j \pi}{3}\right) \operatorname{cis}\left(\frac{\theta}{3}\right)
\end{aligned}
$$

with $j=0,1,2$. If we let

$$
\omega=\operatorname{cis} \frac{2 \pi}{3}=-\frac{1}{2}+\mathrm{i} \frac{\sqrt{3}}{2}
$$

then

$$
\omega^{2}=\operatorname{cis} \frac{4 \pi}{3}=-\frac{1}{2}-i \frac{\sqrt{3}}{2}
$$

and $\omega^{3}=1$. Consequently, for the powers of $\omega$ has a cyclic order of three. Based on this notation, we tabulate the cube roots of $x$ as

$$
\begin{aligned}
& x_{1}=\sqrt[3]{r} \operatorname{cis} \theta \\
& x_{2}=\sqrt[3]{r} \omega \operatorname{cis} \theta \\
& x_{3}=\sqrt[3]{r} \omega^{2} \operatorname{cis} \theta
\end{aligned}
$$

Thus, we found a way to compute cube roots of a number $a_{0} \in \mathbb{C}$ by solving equations in the form $x^{3}-a_{0}=0$.

We proceed solving Eq. 15. Although we seem to have six solutions for Eq. 15, in turn for the resolvent cubic equation, we know this is not the case by Theorem 2 . Thus, we need to make cleaning for obtaining the correct roots. We begin by presenting a condition on the roots of the resolvent cubic equation, which we will use to determine the correct roots.

Lemma 1. If $u_{1}^{3}$ and $u_{2}^{3}$ be two roots of Eq. 15, then $u_{1} u_{2}=-g / 3$.

Proof. Suppose $u_{1}^{3}$ and $u_{2}^{3}$ are roots of Eq. 15 . Then, we can write $\left(u-u_{1}^{3}\right)\left(u-u_{2}^{3}\right)=0$. Hence, we expand to $u^{6}-\left(u_{1}^{3}+u_{2}^{3}\right) u^{3}+u_{1}^{3} u_{2}^{3}=0$, thus, comparing to Eq. 15, we must have $u_{1}^{3} u_{2}^{3}=$ $-g^{3} / 27$. Taking cube roots of both sides, we obtain $u_{1} u_{2}=-g / 3$.

Taking the cube roots of $u_{1}^{3}$ and $u_{2}^{3}$ in Eq. 16, we obtain three roots of $u_{1}^{3}$ as $u_{1}, \omega u_{1}$, and $\omega^{2} u_{1}$. Similarly, three cube roots of $u_{2}^{3}$ are $u_{2}, \omega u_{2}$, and $\omega^{2} u_{2}$. We need to select from each of the three cube roots of $u_{1}^{3}$ and $u_{2}^{3}$ to obtain the roots of the depressed cubic polynomial equation. For this purpose, we present Theorem 3 .

Theorem 3. Let $u_{1}, \omega u_{1}, \omega^{2} u_{1}$ and $u_{2}, \omega u_{2}, \omega^{2} u_{2}$ be roots of Eq. 15. We compute the zeros of the 
depressed resolvent cubic polynomial in Eq. 13 by choosing one from each set of the roots according to the following cases

1. If we choose roots sucht that $u_{1} u_{2}=-g / 3$, then $t_{1}=u_{1}+u_{2}, t_{2}=\omega u_{1}+\omega^{2} u_{2}$, and $t_{3}=\omega^{2} u_{1}+\omega u_{2}$.

2. If we choose roots such that $\omega u_{1} u_{2}=-g / 3$, then $t_{1}=u_{1}+\omega u_{2}, t_{2}=\omega u_{1}+u_{2}$, and $t_{3}=\omega^{2} u_{1}+\omega^{2} u_{2}$.

3. If we choose roots such that $\omega^{2} u_{1} u_{2}=-g / 3$, then $t_{1}=u_{1}+\omega^{2} u_{2}, t_{2}=\omega u_{1}+\omega u_{2}$, and $t_{3}=\omega^{2} u_{1}+u_{2}$.

Proof. We find the roots of the depressed resolvent cubic polynomial by transforming back from $u$ to $t$ by means of the equation

$$
t=u-\frac{g}{3 u}
$$

We can arbitrarily take the cube roots of $u_{1}$ to back substitute for $u$. We can verify that if we choose cube roots of $u_{2}$ instead, we would obtain the same results.

As a consequence of Lemma 1, we need to make sure compatible roots of Eq. 15 are chosen. The possibilities are $u_{1} u_{2}=\omega^{3} u_{1} u_{2}=-g / 3$, $\omega u_{1} u_{2}=\omega^{4} u_{1} u_{2}=-g / 3$, and $\omega^{2} u_{1} u_{2}--g / 3$. Therefore, we consider the following cases

1. If $u_{1} u_{2}=-g / 3$, then the back transformation equation becomes

$$
t=u+\frac{u_{1} u_{2}}{u}
$$

Thus, for $u=u_{1}$, we obtain

$$
t_{1}=u_{1}+u_{2}
$$

Similarly, $u=\omega u_{1}$ would imply

$$
t_{2}=\omega u_{1}+\frac{u_{1} u_{2}}{\omega u_{1}}=\omega u_{1}+\omega^{2} u_{2}
$$

and $u=\omega^{2} u_{1}$ leads to

$$
t_{3}=\omega^{2} u_{1}+\frac{u_{1} u_{2}}{\omega^{2} u_{1}}=\omega^{2} u_{1}+\omega u_{2}
$$

Hence, we obtain the solution set in case 1 of the theorem.
2. If $\omega u_{1} u_{2}=-g / 3$, then the back transformation equation becomes

$$
t=u+\frac{\omega u_{1} u_{2}}{u}
$$

Thus, for $u=u_{1}$, we obtain

$$
t_{1}=u_{1}+\omega u_{2}
$$

Similarly, $z=\omega u_{1}$ would give us

$$
t_{2}=\omega u_{1}+u_{2}
$$

and $u=\omega^{2} u_{1}$ leads to

$$
t_{3}=\omega^{2} u_{1}+\frac{\omega u_{1} u_{2}}{\omega^{2} u_{1}}=\omega^{2} u_{1}+\omega^{2} u_{2}
$$

Thus, $t_{1}, t_{2}, t_{3}$ gives the solution set obtained in case 2 of the theorem.

3. If $\omega^{2} u_{1} u_{2}=-g / 3$, then the back transformation equation becomes

$$
t=u+\frac{\omega^{2} u_{1} u_{2}}{u}
$$

Thus, for $u=u_{1}$, we obtain $t_{1}=u_{1}+\omega^{2} u_{2}$. Similarly, $u=\omega u_{1}$ and $u=\omega^{2} u_{1}$ would give us $t_{2}=\omega u_{1}+\omega u_{2}$ and $t_{3}=\omega^{2} u_{1}+u_{2}$, respectively. Hence, we obtained the solution set presented in case 3 of the theorem.

Thus, for arbitrary selection of two of the cube roots from $u_{1}, \omega u_{1}, \omega^{2} u_{1}$ and $u_{2}, \omega u_{2}, \omega^{2} u_{2}$, we can calculate the roots of Eq. 13 .

Thus, once we obtain $t_{i}, i=1,2,3$ using theorem 3, We can back substitute for $z=t+q / 3$ to obtain the three zeros $z_{i}$ of the resolvent cubic polynomial in Eq. 12.

\subsection{Solving the Quartic Polynomial}

In this section, we present the formulation for finding the zeros of the general quartic monic polynomial in Eq. 2.

We already solved the resolvent cubic polynomial equation to obtain the roots $z_{i}$ which would complete the right hand side of Eq. 6 to a square 
in the form $(m y+k)^{2}$ with $m$ and $k$ expressed in Eq. 8 and Eq. 9, respectively. Consequently, we can write the general quartic polynomial equation in the form

$$
\left(y^{2}+\frac{1}{2} z_{i}\right)^{2}=\left(m_{i} y+k_{i}\right)^{2}
$$

with $z_{i}$ being a root of the resolvent cubic polynomial equation and $m_{i}, k_{i}$ being the values evaluated for the root $z_{i}$. Since we have three roots from the resolvent cubic polynomial, it would be a natural question to choose which one for $z_{i}$. We discussed in Section 2 that a quartic polynomial has exactly four zeros in $\mathbb{C}$. Hence, we choose one of the three roots of the resolvent polynomial equation arbitrarily [2] while neglecting the others.

Selecting $z_{i}$, we can take the square roots of both sides of Eq. 21. Thus, we obtain the following two quadratic equations

$$
\begin{aligned}
& y^{2}-m_{i} y-k_{i}+\frac{1}{2} z_{i}=0 \\
& y^{2}+m_{i} y+k_{i}+\frac{1}{2} z_{i}=0
\end{aligned}
$$

The zeros of the first quadratic polynomial give us two of the roots of the depressed quartic polynomial equation

$$
y_{1}=\frac{m_{i}}{2}-\sqrt{\frac{m_{i}^{2}}{4}+k_{i}-\frac{z_{i}}{2}}
$$

and

$$
y_{2}=\frac{m_{i}}{2}+\sqrt{\frac{m_{i}^{2}}{4}+k_{i}-\frac{z_{i}}{2}}
$$

while that of the second quadratic polynomial give the other two roots of the depressed quartic polynomial equation

$$
\begin{aligned}
& y_{3}=-\frac{m_{i}}{2}-\sqrt{\frac{m_{i}^{2}}{4}-k_{i}-\frac{z_{i}}{2}} \\
& y_{4}=-\frac{m_{i}}{2}+\sqrt{\frac{m_{i}^{2}}{4}-k_{i}-\frac{z_{i}}{2}}
\end{aligned}
$$

Once we obtain the roots of the depressed quartic equation, we can transform to obtain the roots of Eq. 2 by using $x_{i}=y_{i}-b_{3} / 4$. This completes the formulation to obtain the roots of a general quartic polynomial equation.

\section{Case Study: Self-Reciprocal Quartic Polynomials}

We formulated a solution algorithm to obtain roots of the general quartic polynomial equation in Section 3. The solution included solving a cubic resolvent of the quartic polynomial. Although, solving a cubic polynomial equation is necessary for the general quartic case, the method would be simpler if we consider specialization of the general quartic polynomial. One such specialization is a self-reciprocal quartic polynomial, which we study in this section. Under a suitable transformation, solution of self-reciprocal quartic polynomial equations reduce to solving two quadratic polynomials.

Definition 1 (Self-Reciprocal Quartic Polynomial). Let $F$ be a subfield of the field of the real numbers $(\mathbb{R})$. A quartic polynomial $f \in F[x]$ given by the equation 1 is self-reciprocal under the condition that $f(x)=f^{\dagger}(1 / x)$ with $f^{\dagger} \in F[x]$ defined as:

$f^{\dagger}(x)=x^{4} f\left(\frac{1}{x}\right)=c_{0} x^{4}+c_{1} x^{3}+c_{2} x^{2}+c_{3} x+c_{4}$

Thus, $c_{0}=c_{4}$ and $c_{1}=c_{3}$.

Thus, we can write a general quartic selfreciprocal polynomial equation as:

$$
c_{0} x^{4}+c_{1} x^{3}+c_{2} x^{2}+c_{1} x+c_{0}=0
$$

Without loss of generality, we can divide both sides by $c_{0}$ to obtain

$$
x^{4}+d_{1} x^{3}+d_{2} x^{2}+d_{1} x+1=0
$$

with $d_{1}=c_{1} / c_{0}$ and $d_{2}=c_{2} / c_{0}$. The roots of the self-reciprocal polynomial equation are a consequence of inversion with respect to the unit circle [3]; thus, the roots are never equal to zero. Using this knowledge, we can divide both sides of Eq. 28 by $1 / x^{2}$ to obtain

$$
x^{2}+d_{1} x+d_{2}+d_{1} \frac{1}{x}+\frac{1}{x^{2}}=0
$$


Let us define $v=x+1 / x$, which is a transformation discussed in [6]. Then, collecting the terms with the same coefficient in Eq. 29, we obtain

$$
x^{2}+d_{1} v+d_{2}+\frac{1}{x^{2}}=0
$$

We can take squares of the transformation for $v$ and rearrange to obtain

$$
x^{2}+\frac{1}{x^{2}}=v^{2}-2
$$

Substituting above into Eq. 30, we write

$$
v^{2}+d_{1} v+d_{0}=0
$$

with $d_{0}=d_{2}-2$. The roots of the quadratic equation are

$$
\begin{aligned}
& v_{1}=-\frac{d_{1}}{2}-\sqrt{\frac{d_{1}^{2}}{4}-d_{0}} \\
& v_{2}=-\frac{d_{1}}{2}+\sqrt{\frac{d_{1}^{2}}{4}-d_{0}}
\end{aligned}
$$

We can transform back $v_{i}=x+1 / x$ with $i=1,2$ to obtain the quadratic equation

$$
x^{2}-v_{i} x+1=0
$$

Solving this quadratic equation gives us the roots of the quartic self-inversive polynomial as

$$
\begin{aligned}
& x_{1}=\frac{v_{1}}{2}-\sqrt{\frac{v_{1}^{2}}{4}-1} \\
& x_{2}=\frac{v_{1}}{2}+\sqrt{\frac{v_{1}^{2}}{4}-1} \\
& x_{3}=\frac{v_{2}}{2}-\sqrt{\frac{v_{2}^{2}}{4}-1} \\
& x_{4}=\frac{v_{2}}{2}+\sqrt{\frac{v_{2}^{2}}{4}-1}
\end{aligned}
$$

Thus, we can obtain the roots of a self-reciprocal quartic equation without having to solve a resolvent cubic polynomial equation.

\subsection{Example}

In this section, we solve a self-reciprocal quartic polynomial which is available in [3]. The polynomial is below

$$
x^{4}-\frac{213}{50} x^{3}+\frac{165857}{25600} x^{2}-\frac{213}{50}+1=0
$$

We will solve this equation by using the method discussed in this section, then we will solve it by considering it as a general quartic polynomial. We will be writing the radical expressions in decimal form to save space when necessary.

\section{Solution 1}

Comparing the example with Eq. 28, we find $d_{1}=-213 / 50$ and $d_{2}=165857 / 25600$. Transforming this equation by the method discussed in this section, we obtain $d_{0}=114657 / 25600$. Thus,

$$
v^{2}-\frac{213}{50} v+\frac{114657}{25600}=0
$$

Hence, we obtain $v_{1}=2.37106$ and $v_{2}=1.88894$ by using Eq. 32. Then, we find the zeros of the self-reciprocal polynomial from Eq. 34 as $x_{1}=0.548755, x_{2}=1.82231, x_{3}=0.944469-$ $\mathrm{i} 0.328601$, and $x_{4}=0.944469+\mathrm{i} 0.328601$.

\section{Solution 2}

We use the formulation for the general quartic polynomial in Section 3 to obtain the same solution.

We obtain the depressed quartic polynomial equation by using the transformation $x=y+$ 213/200. Using Eq. 4, we compute $q=$ $-208999 / 640000, r=-7921683 / 64000000$, and $s=-1226099903 / 25600000000$. Thus, we write

$y^{4}-\frac{208999}{640000} y^{2}-\frac{7921683}{64000000} y-\frac{1226099903}{25600000000}=0$

We proceed with obtaining the resolvent cubic polynomial by using Eq. 12 as

$$
\begin{gathered}
z^{3}+\frac{208999}{640000} z^{2}+\frac{1226099903}{6400000000} z \\
+\frac{12093787004663}{256000000000000}=0
\end{gathered}
$$


Making another transformation, which would be $z=t-208999 / 1920000$, we compute $g=306768959 / 1966080000$ and $h=$ $6560810064991 / 226492416000000$ by using Eq. 15. Thus, we obtain the depressed resolvent cubic as

$$
t^{3}+\frac{306768959}{1966080000} t+\frac{6560810064991}{226492416000000}=0
$$

Then, we use Eq. 16 to obtain

$$
\begin{aligned}
u_{1}^{3}= & -\frac{86779}{1638400000} \sqrt{\frac{1873891}{15}} \\
& -\frac{6560810064991}{452984832000000} \\
= & -0.0332042
\end{aligned}
$$

and

$$
\begin{aligned}
u_{2}^{3}= & \frac{86779}{1638400000} \sqrt{\frac{1873891}{15}} \\
& -\frac{6560810064991}{452984832000000} \\
= & 0.00423715
\end{aligned}
$$

We will present the rest of the solution in decimal form to save space. We note that $u_{1}^{3}=$ $0.0332042 \operatorname{cis} \pi$ and $u_{2}^{3}=0.00423715$ cis0. Thus, we can calculate the cubic roots of $u_{1}^{3}$ and $u_{2}^{3}$ by using Eq. 20. If we pick $u_{1}=0.160707+$ $0.278352 i$ and $u_{2}=0.161817$, then we obtain

$$
\omega u_{1} u_{2}=-0.0520103-\mathrm{i} 3.72529 \times 10^{-9}
$$

which is equivalent to $-g / 3=-0.0520103$ if we discard Sagemath numerical errors. Thus, we can use the case 2 of Theorem 3 to obtain $t_{1}, t_{2}, t_{3}$. Substituting the values back by using

$$
z_{i}=t_{i}-\frac{208999}{1920000} \quad i=1,2,3
$$

Thus, we obtain the roots of the resolvent cubic polynomial equation as

$$
\begin{aligned}
& z_{1}=-0.0290555+\mathrm{i} 0.418490 \\
& z_{2}=-0.268450-\mathrm{i} 2.98023 \times 10^{-8} \\
& z_{3}=-0.0290554-\mathrm{i} 0.418490
\end{aligned}
$$

To continue solution of the quartic polynomial equation, we arbitrarily pick $z_{1}$ and calculate $m=$ $0.636776+\mathrm{i} 0.328601$ and $k=0.0767513-$ i0.0396066 by using Eq. 8 and Eq. 9, respectively. We then use Eq. 23 through Eq. 26 to find the zeros of the depressed quartic polynomial, and back substitute for $x_{i}$

$$
x_{i}=y_{i}+\frac{213}{200} \quad i=1, \ldots, 4
$$

to obtain

$$
\begin{aligned}
& x_{1}=0.944469+\mathrm{i} 0.328601 \\
& x_{2}=1.82231 \\
& x_{3}=0.548755+\mathrm{i} 1.49012 \times 10^{-8} \\
& x_{4}=0.944469-\mathrm{i} 0.328601
\end{aligned}
$$

Neglecting the small imaginary number in $x_{3}$ as a consequence of the Sagemath computational errors, the results $x_{i}$ are in agreement with that the solution method 1 and also with the result available in [3].

\section{Conclusion}

As we have studied, solving general quartic polynomial equations by radicals is straightforward by means of a few transformations, so the technique does not require much computational effort. This would allow us to do the computations with relative ease instead of trying a more sophisticated algorithm.

As we studied in Section 4.1, the formulation for solving the general quartic polynomial equations can be used for specialized polynomials such as self-reciprocal polynomials. However, a selfreciprocal quartic polynomial can be transformed into a simpler form which does not require solution of a cubic polynomial equation, thus make the solution even easier to implement. We illustrate the ease of solution of a self-reciprocal quartic polynomial equation by means of a suitable transformation $x+1 / x$ in solution 1 of the example in section 4 . 


\section{References}

[1] I. Stewart, Galois Theory. CRC Press, Boca

Raton, FL, fourth ed., 2015.

[2] B. M. Kiernan, "The Development of Galois Theory from Lagrange to Artin," Archive for History of Exact Sciences 8 (December, 1971) 40-154.

[3] S. Sahin, "Solving the Forward Position Problem of an In-Parallel Planar Manipulator in the Gauss Plane," ArXiv e-prints (Nov., 2015), arXiv:1511.05060 [CS.RO].

[4] T. W. Judson, Abstract Algebra. No. 3 in Mathematics Textbook Series. Virginia Commonwealth University, Richmond, VA, 2011. http:

//abstract.ups.edu/index.html.

[5] R. Irving, Beyond the Quadratic Formula. Classroom resource materials. Mathematical Association of America, 2013.

[6] D. E. Littlewood, The Skeleton Key of Mathematics. Dover Publications, Mineola, NY, 2002. 